\title{
Clustering based Load Balanced Gateway Placement Approach
}

\author{
Smriti Srivastava \\ Amity University, Noida
}

\author{
Anant Kumar Jaiswal \\ Assistant Professor \\ Amity University, Noida
}

\begin{abstract}
Wireless mesh networks (WMN) is a promising technology to provide broadband Internet access. Gateway placement is an important part of WMN design that determines how to place the gateways in WMN. Various approaches have been given by various researchers for Gateway placement taking care of various parameters. In this paper, we proposed a clustering based load balanced algorithm to find an approximating optimal solution of gateway placement in WMN. The aim of our research is to focus on load balanced gateway placement problem.
\end{abstract}

\section{Keywords}

Wireless mesh network; gateway placement; load balanced.

\section{INTRODUCTION}

With the incredible success of wireless technologies and the high demand of Internet Access, the design of cost-effective and scalable wireless networks is becoming necessary. In the current scenario, everything is connected throughout the world by a network; Wireless network communication has gained much popularity last decades. Wireless networks do not need a wired backbone because of this they become a good option for latest network situations. For next-generation wireless networking, WMNs which are undergoing rapid progress and inspiring many applications have emerged as a key technology but still many issues exist in this field. The WMN nodes are comprised of mesh routers (MRs), gateways (GWs) and mesh clients (MCs).MRs provide wireless connections for MCs as access point in their respective areas and acts as a wireless backbone; also they relay traffic for other MRs. Some MRs called gateways is directly connected to the Internet via physical link. In WMN, gateways are special MRs configured with wired links, which act as the bridges between the WMN and the Internet. MCs are mobile clients, e.g., desktops, laptops, PDAs and phones. Each MC associates with one of the nearest MRs, and accesses the Internet by wireless multi-hop forwarding. Akyildiz et al. [5] presented a survey of WMNs, and discussed some research issues. Gateway placement which means to find the optimal number and location of gateways is an important and challenging issue. It determines how many gateways are needed and where they should be placed. In order to improve throughput more gateways can be added and selecting appropriate gateway locations can also optimize network topology and traffic distribution. But, due to costly construction of wired links in gateways, adding more gateways will increase the cost.

\section{Issues related to gateway placement:}

i) Bandwidth: Bandwidth is a rate of data transfer, measured in bits per second. While placing the gateways, one must keep in mind that the Placement of the gateways should satisfy the bandwidth requirement of all the clients.

ii) Congestion: placement of gateways should be done in such a way that node is not over congested and load should be properly balanced.

iii) Cost: Adding more number of gateways will increase network throughput but it results in higher cost of construction of wired links. So it is necessary to minimise the number of gateways and still do not compromise on throughput or delay.

iv) Interference: interference among gateways should be minimum and throughput should be maximum.

v) Distance: Interference among gateways will degrade network throughput if gateways are placed densely and if the distance between the gateway is too much then the signal strength is degraded. So distance between gateways should be optimal.

vi) Delay: If many intermediate nodes come in transmission path, then the frequent storage and transmission on intermediate nodes would add the transmission delay time, and the efficiency of communication will reduce. So, placement of gateways must be done while keeping in mind that the delay in transmission should be minimum.

vii) Location: If gateways are located densely then interference increases thus enhancing the cost of setup otherwise it may lead to poor signal strength. So determining a proper location of gateways is another issue.

vii) Coverage: It means number of nodes served by each gateway. Each Mesh Routers must be covered by more than one gateway so that if one of the gateways fails the MRs may be covered by the backup gateway.

Gateway placement is an important part of WMN design that determines how to place the gateways in WMN. Placing too many Gateways will, on one side improve the throughput and reduces congestion but on the other side increase interference and cost. So the various approaches have been given by various researchers for Gateway placement taking care of various parameters important in Backbone network. This research will emphasis on placing a gateway in WMN and no Gateway is overloaded at any point of time 


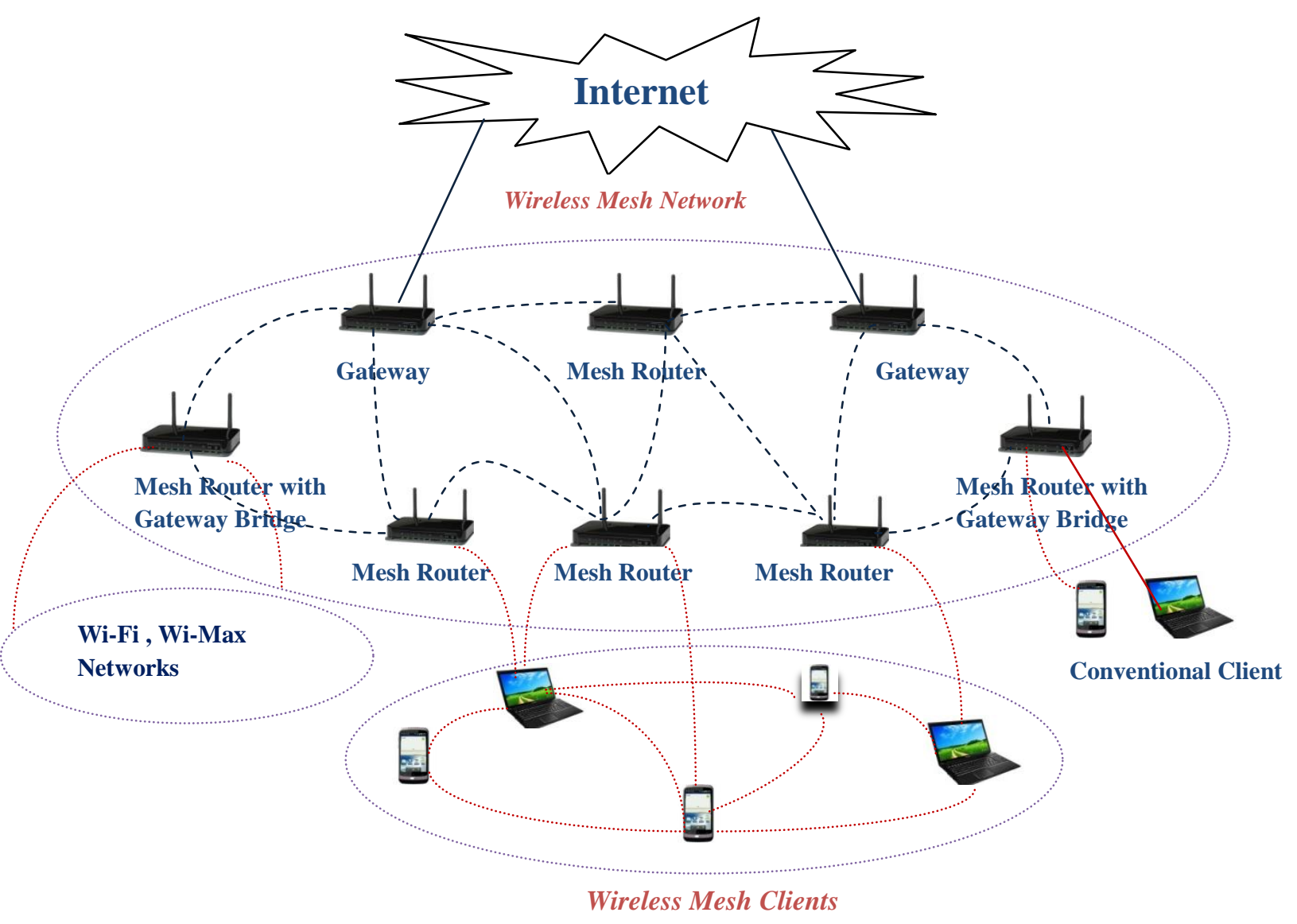

Fig1: Wireless Mesh Infrastructure

\section{RELATED WORK}

Much research work has been done regarding the placement of gateway in wireless mesh networks (WMNs).

A popular approach is to divide the network into clusters, and a single mesh router is chosen as the gateway to within the cluster Research in [2-4] and [16] has used different clustering-based approaches. Y. Drabu et al. [2] addressed gateways placement problem, consisting of placing a minimum number of gateways so that quality-of-service (QoS) requirements are satisfied and also presented a near optimal heuristics algorithm for gateway placement. B. Aoun et al. [3] addressed the problem of gateways placement by placing a minimum number of gateways such that quality-ofservice (QoS) requirements are satisfied using polynomial time near-optimal algorithm. F. Zeng et al. [4] addressed the problem of load balanced gateway placement, and proposed a greedy algorithm GA-LBC to partition a WMN into loadbalance and disjointed clusters, each cluster satisfies QoS requirements. In paper [16] author addressed the gateway placement problem as a variant of the capacitated facility location problem (CFLP), and proposed a clustering algorithm. Each gateway served a cluster of its nearby MRs, and a spanning tree rooted at the gateway (cluster head) was used for message delivery.

Different gateway placement approaches have also been proposed in [10-12] for network throughput optimization. In paper [10] an innovative gateway placement approach was proposed for (WMNs) in which the proposed gateway placement scheme provides a framework of maximizing the throughput of WMNs through proper placement of these gateways. F.Li et al. [11] addressed the problem of gateway placement for throughput optimization in multi-hop wireless mesh networks and also proposed a novel grid-based gateway deployment method.Q. Xin et al. [12] have studied the problem of gateway selection for throughput optimization in multi-radio multi-channel wireless mesh networks. He also proposed a new gateway deployment method using a cross layer throughput optimization, Z.Hu et al.[18] proposed a heuristic gateway placement algorithm using directional antenna in order to minimize the total number of gateways and the average and maximum hop count between any mesh router and its closest gateway. Wireless links in directional BWMNs are point-to-point directional links. Interference is greatly reduced because of the use of directional antennas. Here proposed algorithm operates as follows: In step 1 node with the highest capacity, is chosen as a gateway. At step 2 a minimum distance $(\mathrm{h})$ is set to make sure that the gateways are kept apart from each other. Step 3 assures that the least number of gateways is set to meet the total traffic demand. Multiple Antenna based fault tolerant approach is better because it considered fault-tolerance, throughput as well as cost. 
S. Ivanovo et al. [19] papers approach is to extend the existing methods from infrastructure network planning to plan the multi-hop wireless mesh networks with fault-tolerance aspects. The base station planning algorithm provides coverage in a predefined area by adjusting the number and the position of the base stations. But this approach also works for limited number of faults that is can work only for one base station failure.

Chandra et al. [20] addressed the gateway placement problem aiming to minimize the number of gateways while satisfying bandwidth requirements of all clients. They defined the wireless link models, and developed gateway placement algorithms for each model. The gateway placement problem was formulated as a network flow problem, and a max-flow min-cut based algorithm was developed for gateway selection

\section{PROPOSED MODEL}

The main focus of this paper is on the problem of load balanced gateway placement. The overall cost of placing a gateway is thousand times higher than a normal mesh router; so, to minimize the total cost, the number of deployed gateways should be kept as low as possible. The gateway should be selected in such a manner that it always satisfies the condition that total capacity of all the gateways should be at least total traffic demand imposed by all the clients; to minimize the number of gateways, a mesh router with higher capacity should have higher priority of being selected as a gateway. Here, the capacity of a node refers to its capacity of handling local traffic plus its backbone traffic.

By adding more gateways network throughput can be increased by effectively reducing the average minimum hop count that a message has to go through to reach the nearest gateway, and alleviating traffic load on existing gateways. In addition, the locations of deployed gateways can affect network performance greatly. For example, more gateways need to be placed in the areas of heavy traffic load, so that traffic load balancing can be achieved among all gateways. The locations of deployed gateways determine the minimum hop count for any mesh router to reach the nearest gateway. It is obvious that the nodes of higher degree are easy for more nodes to reach in relatively short hop counts. Based on above analysis, the following factors are to be considered in the work.

- In order to minimize the cost, the number of gateways should be kept as low as possible.

- The traffic demand can be handled directly without occupying the backbone bandwidth only when the gateway is chosen from among nodes of heavy local traffic demand.

- While placing a gateway hop counts should be maintained in such a manner so that mesh routers at different areas can reach respective nearest gateway in short hop count.

- A gateway node should have high backbone bandwidth so that it can handle more backbone traffic that can be delivered to or from the Internet

\subsection{Assumption}

- Let the backbone of BS be a connected graph of base stations, where BS can be speculated as nodes and the edge in the graph will work like the links between the nodes .So we use an undirected connected graph $\mathrm{G}(\mathrm{V}, \mathrm{E})$ to represent WMN backbone network, in which an edge represents a communication link connecting two nodes and a vertex represents a mesh router (or nodes). $\mathrm{V}$ is set of all Vertices (or nodes).E is a set of all edges (or links).We assume that the initial graph G, Compromised of MR nodes, is connected.

- To determine the minimum degree of the graph, we use a property called bi connectivity in a network. We can obtain the minimum degree of all the nodes in the graph. (The minimum degree of the graph is the number of incoming and outgoing links of the node).Each node should be covered by at least two base stations. So the minimum degree of 2 should be maintained in the resultant graph for all the nodes.

\subsection{Overview of Algorithm}

In this paper a load balanced algorithm to find an approximating optimal solution of gateway placement in WMN is proposed. This algorithm is separated into two steps:

\section{Candidate MG Position}

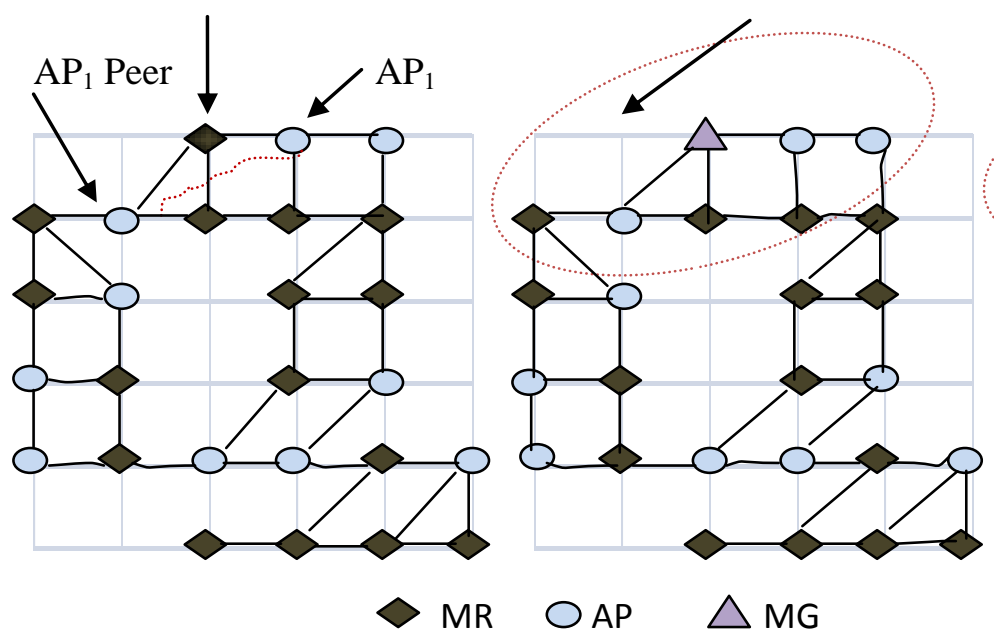

The First Cluster is Formed Special Case of One AP Left

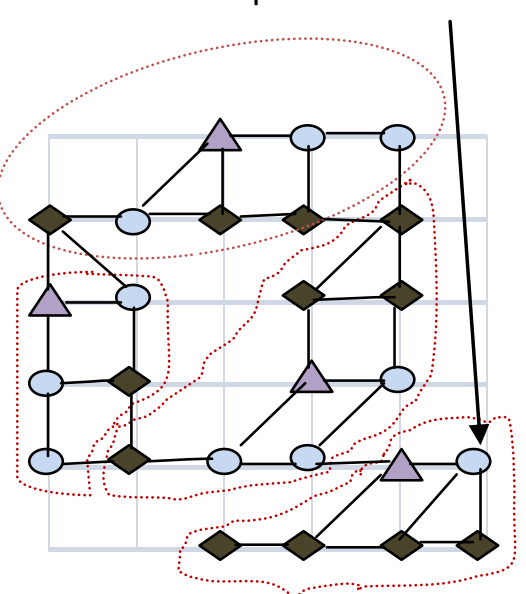

Fig2:Different Stages in clusters construction and gateway placement on $6 \times 6$ grid based $\mathrm{WMN}$ with $\mathrm{R}=3$ and $\mathrm{H}=2$ 
In the first step an approach of gateway selection, which is similar to finding minimum dominating set with maximal weight is presented, and in second step, every MR can be attached to one or more gateways. In order to determine which routers should be selected as gateways under connectivity, bounded delay, and financial constraints one should firstly focus on the problem that the location of APs and MRs with their traffic demands are given. For this a clustering based gateway placement approach is proposed which aims at: (1) Ensuring the strategic placement of MGs. On the basis of cluster radius and expected path length from the selected MG to all APs belonging to the same cluster the position of a candidate MG is selected;(2) Network scalability by forcing each node to send traffic to its closest gateway is assured; (3) Placement of a smaller number of MGs, which resulting in cost effective topologies. This technique ensures a proper placement of MGs which lead to less deployment cost while providing enough network throughput capacity.

First of all the set of APs is defined and the whole graph should be fully connected, thereafter cluster construction procedure starts by placement of a MG at a half way position between two APs. For the AP which is not yet visited, its peer AP is selected as the closest one, among its entire AP neighbour (within single hop distance) is not considered as candidate APs. Here Best First Search algorithm for the selection of the peer AP is used, since all graph links have the same cost (equal to one). The set of mesh nodes that are at most $\mathrm{H}$ hops away from the newly placed MG are included in the same cluster and marked as visited. The algorithm is iterative and terminates when all mesh nodes are marked. When there are one or two AP left (no peer AP is found), there parent in the routing path (its predecessor) is selected as MG in that situation; all the nodes belong to disjoint clusters, each headed by one MG when the algorithm terminates. The total number of formed clusters represents then, the minimal number of MGs that assures the required delay with a minimum deployment cost.

The proposed gateway placement scheme operates as follows.

Step1: Begin with the node having highest capacity, and set it as a gateway.

Step2: Discover mesh routers that are at least $\mathrm{h}$ hops away from all existing gateways. Set the node of highest capacity as a gateway.

Step3: If the total capacity of all existing gateways does not exceed the total traffic demand, repeat step 2 until all traffic demands is considered.

As Step1, for nodes of same backbone bandwidth, those of heavy local traffic have higher probability of being chosen as a gateway. At step2, a minimum distance (h) is set to ensure that the gateways are kept apart from each other. An optional value of $\mathrm{h}$ can be found depending on network size

\subsection{Proposed Algorithm}

Initialize $\mathrm{j}$ to zero;

Set Ltc=APnum;

Set $t \_A p \_c=0$;

ShortestPathLength $=\infty$;

While $(\mathrm{j}<$ APnum) \&\& (Ltc $>1)$ do

While (exist $\mathrm{k}$ belongs to list of non clustered APs) do

If $\mathrm{APj}$ and $\mathrm{APk}$ are $\mathrm{H}+1$ hop apart then pathLength $=$ BestFirstSearch $(A P j, A P k)$

If the pathLength $<$ shortestPathLength then set index $=k$;
End if

$k=k+1$;

End if

End while

Set Flag $(A P j)=1$;

Set Flag $($ APindex $)=1$;

Increment t_Ap_c by 2 ;

$\mathrm{Ltc}=$ APnum- t_Ap_c;

G_index= GoBackHalfWay $(A P j$, APindex $)$;

Set Gateway [G_index] to 1;

ConstructOneCluster (G_index, $\mathrm{H})$;

Update (t_Ap_c);

$\mathrm{j}=\mathrm{j}+1$;

End while

If $\mathrm{Ltc}=1$ then

Set $G_{-}$index to index of pathParent of AP left;

Set Gateway [G_index] to 1 ;

End if

Pseudo code for ConstructOneCluster: All mesh nodes that are located within a radius of $H$ hops from $\mathrm{G}_{-}$index are included in the same cluster (ConstructOneCluster ()). APs included in that cluster are marked and the total number of clustered APs is then updated (Update ()).
Initialize t_Ap_c to 0;
Initialize i to 0 ;
Run while loop until $\mathrm{i}<=\mathrm{H}$
Increment t_Ap_c by 1 ;
Increment $\mathrm{i}$ by 1 ;
End While

Pseudo Code for GoBackHalfWay: GoBackHalfWay () returns the position of the candidate MG, G_index, which is equidistantly located between $A P j$ and APindex

Initialize $\mathrm{i}$ to 0 ;

Set $\mathrm{K}=(\mathrm{APj}+\mathrm{APindex}) / 2$;

Return k.

Here Ltc denotes the nodes that are left to be clustered and t_Ap_c denotes the total number of AP clustered.

Lemma: At the end of clustering process, each cluster is headed by one $M G$.

PROOF: Assume that a cluster is formed without placing an MG at the centre of the cluster as cluster head. This implies that Go Back Half-Way $(A P j, A P$ index) does not return any node to be elected. In this case, executing Best First Search $(A P j, A P k)$ would not find a path between $A P j$ and $A P k$ which later becomes APindex as the closest peer. This means that $A P j$, and APindex belong to two disconnected graphs whereas the graph is connected. This proves that our assumption is false; thus, Lemma.

\section{CONCLUSION AND FUTURE WORK}

As the problem of Gateway placement plays a vital role in determining the throughput of the network, an optimum gateway placement algorithm is proposed which shares the load equally among various nodes. In future we would make it 
fault tolerant as well and through proper simulation model we will prove that our algorithm is better than others.

\section{REFERENCES}

[1] D. Benyamina, A. Hafid, and M.Gendreau, "Optimal Placement of Gateways in Multi-hop Wireless Mesh Networks: A Clustering based Approach," IEEE 34thConference on Local Computer Networks (LCN2009), pp.625-632, 2009.

[2] Y. Drabu and H. Peyravi, "Gateway Placement with QoS Constraints in Wireless Mesh Networks," in IEEE 7th International Conference on Networking, 2008, pp. 4651.

[3] B. Aoun, R. Boutaba, Y. Iraqi, and G. Kenward, "Gateway Placement Optimization in Wireless Mesh Networks With QoS Constraints," IEEE Journal on Selected Areas in Communications, Vol. 24, No. 11, pp. 2127-2136, 2006.

[4] F. Zeng and Z. Chen, "Load Balancing Placement of Gateways in Wireless Mesh Networks With QoS Constraints," IEEE 9th International Conference for Young Computer Scientists, ICYCS 2008, pp. 445-450.

[5] I. F. Akyildiz, X. Wang, and W. Wang, "Wireless mesh networks: A survey," Computer Networks, vol. 47, no. 4, pp. $445-487,2005$

[6] Y. Zhang, J. Luo, and H. Hu, Wireless mesh networking: architectures, protocols and standards. Auerbach Pub, 2006.

[7] lunzhau Lua, Wenjia Wu, Ming Yang" Interferenceaware Gateway Placement for Wireless Mesh Networks with Fault Tolerance Assurance".

[8] S. Waharte, R. Baouf, and P. Anelli, "Impact of Gateways Placement on Clustering Algorithms in Wireless Mesh Networks," IEEE International Conference on Communications, ICC 2009, pp.1-5.

[9] W. Wu, J. Luo, and M. Yang, "Gateway Placement Optimization for Load Balancing in Wireless Mesh Networks," Proceedings of the 13th International 179 Conference on Computer Supported Cooperative Work in Design, pp. 408-413, 2009.

[10] P. Zhou, B. S. Manoj, and R. Rao, "A Gateway Placement Algorithm in Wireless Mesh Networks," Proceedings of the 3rd International Conference on Wireless Internet, ACMWICON 2007, pp.1-9.
[11] F. Li, Y. Wang, and X.-Y Li, "Gateway Placement for Throughput Optimization in Wireless Mesh Networks," ACM Mobile Networks and Applications, Vol. 13, Iss. 12, 2008, pp.198-211.

[12] Q. Xin and Y. J. Wang, "Gateway Selection Scheme for Throughput Optimization in Multi-radio Multi-channel Wireless Mesh Networks," The 5th International Conference on Mobile Ad-hoc and Sensor Networks, 2009, pp.187195.

[13] J. W. Tsai and T. Moors, "Minimum Interference Multipath Routing Using Multiple Gateways in Wireless Mesh Networks," IEEE 5th International Conference on Mobile Ad Hoc and Sensor Systems, 2008, pp. 519-520.

[14] P. Jun and Q. Zhou, "Gateway Placement Optimization in Wireless Mesh Networks," IEEE International Conference on Networking and Digital Society, pp. 221 226, 2009.

[15] S. Tajima, T. Higashino, N. Funabiki, and S. Yoshida, "orksAn Internet Gateway Access-point Selection Problem for Wireless Infrastructure Mesh Netw," Proceedings of IEEE 7th International Conference on Mobile Data Management, pp.112-112. 2006

[16] Y. Bejerano, "Efficient integration of multi hop wireless and wired networks with qos constraints," IEEEIACM Transactions on Networking,vol. 12, no. 6, pp. 1064 1078,2004

[17]B.He, B. Xie, and D. P. Agrawal, "Optimizing deployment of internet gateway in wireless mesh networks," Computer Communications, vol. 31, no. 7, pp. 1259 - 1275, 2008.

[18] Ziping Hu, Pramode K. Verma "Gateway Placement in Backbone Wireless Mesh Networks using Directional Antennas", 2011 Ninth Annual Communication Networks and Services Research Conference.

[19] S. Ivanov, E.Nett. And R. Schumann. Fault-tolerant base station planning of wireless mesh networks in Dynamic Industrial Environments. 2010.

[20]R. Chandra,L . Qiu,K . Jain,a nd M. Mahdian," Optimizing the placement of internet taps in wireless neighborhood networks," in Proceedings -International Conference on Network Protocols, ICNP, Berlin,G ermany,2004, pp. 271 - 282. 